\title{
Incessant Monomorphic Ventricular Tachycardia Induced by the Proarrhythmic Effect of Amiodarone
}

\author{
Hisaki Makimoto, Takashi Noda, Takashi Kurita, Ikutaro Nakajima, Teruki Yokoyama, \\ Atsushi Doi, Yuko Yamada, Hideo Okamura, Kazuhiro Satomi, Takeshi Aiba, \\ Wataru Shimizu, Kazuhiro Suyama, Naohiko Aihara and Shiro Kamakura
}

\begin{abstract}
This case report describes incessant monomorphic ventricular tachycardia (VT), not Torsade de Pointes, induced by intravenous amiodarone in a 48-year-old woman with dilated cardiomyopathy. VT was reproducibly triggered by short coupled premature ventricular complex (PVC) with different morphology from VT. After amiodarone infusion, the coupling interval of initiating PVC was prolonged, and moreover, the morphology of initiating PVC became the same as that of VT. Though amiodarone has become the first line drug to treat ventricular tachyarrhythmias in patients with cardiac dysfunction, it is important to be aware of its proarrhythmic effect, which may lead to an electrical storm of monomorphic VT.
\end{abstract}

Key words: monomorphic ventricular tachycardia, amiodarone, proarrhythmia, Torsade de Pointes, class III antiarrhythmic

(Intern Med 50: 2591-2595, 2011)

(DOI: 10.2169/internalmedicine.50.5588)

\section{Introduction}

Ventricular tachycardia/fibrillation (VT/VF) is one of the major causes of death in patients with structural heart disease. Amiodarone, which is classified as a class-III antiarrhythmic agent, is considered as the most efficacious agent even for patients with severe cardiac dysfunction (1). Amiodarone has been broadly used in the emergency department of outpatient clinics, and it is also well known that the electrophysiological effect of amiodarone is different when it is administered orally or intravenously (2). Although proarrhythmic effects of amiodarone are rare, some patients occasionally develop polymorphic VT of Torsade de Pointes (TdP) $(3,4)$. We present an unusual case with incessant monomorphic VT, not TdP, induced after amiodarone infusion.

\section{Case Report}

A 48-year-old woman was admitted to our hospital due to sudden palpitation, and dyspnea. She had been diagnosed as idiopathic dilated cardiomyopathy when she was 24 years old and treated with carvedilol, digoxin, and enalapril. Her family history included dilated cardiomyopathy in her brother, sister, and son. She had been seemingly healthy until the morning of the day of admission. She suddenly recognized palpitation, dyspnea, and cold sweat when she was riding her bicycle. Though those symptoms were relieved with 15 minutes' rest, she felt disturbed pulse. She presented to our emergency department immediately.

She felt no symptom on admission. Chest radiogram showed cardiomegaly. Her electrocardiogram (ECG) showed sinus rhythm of 84 beats/min with occasional premature ventricular complexes (PVCs) (Fig. 1A). After admission, we evaluated her clinical picture. Her echocardiogram showed a markedly dilated left ventricle (Dd/Ds 77/63 mm) with the left ventricular ejection fraction of $17 \%$. Blood tests revealed no significant abnormalities including electrolyte disturbance. Suddenly, recurrent ventricular tachycardia (VT) was observed on her monitoring ECG during the hospitalization (Fig. 1B). There were no significant triggers of

Division of Arrhythmia and Electrophysiology, Department of Cardiovascular Medicine, National Cerebral and Cardiovascular Center, Japan Received for publication April 1, 2011; Accepted for publication July 22, 2011

Correspondence to Dr. Takashi Noda, tnoda@hsp.ncvc.go.jp 

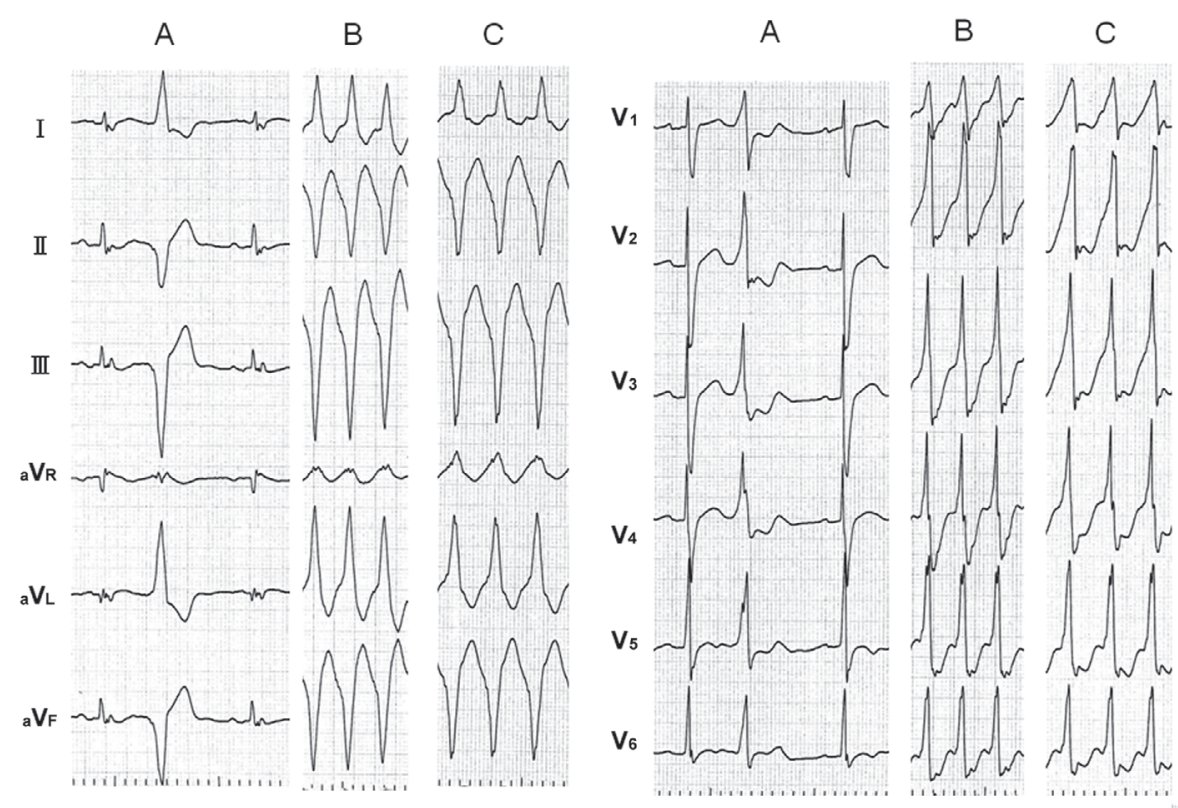

Figure 1. Twelve-lead electrocardiograms (ECGs) on admission and during ventricular tachycardia (VT) before and after amiodarone. The ECG showed sinus rhythm of 84 beats per minute with first degree atrio-ventricular block, and occasional premature ventricular complexes (PVC) (A). Before amiodarone administration, the QRS morphology of incessant VT was right bundle branch block morphology with superior axis (B). After amiodarone administration, the QRS morphology of VT was also similar to that of VT before amiodarone, but VT rate decreased to 150 beats per minute (C).

arrhythmia such as a change in drug dosage, electrolyte disturbance, infection, or exercise. VT rate was 170 beats/min and she complained of palpitation.

After $125 \mathrm{mg}$ of amiodarone was administered intravenously over 10 minutes, continuous intravenous administration (40 mg per hour) was given. An hour after starting amiodarone, the VT rate decreased to 150 beats/min but the duration of VT was still prolonged (Fig. 1C). Moreover, the morphology of the initiating PVC was changed. Before amiodarone, VT was triggered by PVC of which the morphology differed from that of VT (Fig. 2A). After one hour of intravenous amiodarone, the culprit PVC in the baseline was completely eliminated, and then sustained VT was triggered by the PVC, whose morphology was virtually identical to the sustained VT (Fig. 2B).

During sinus rhythm, QRS duration was prolonged from $130 \mathrm{~ms}$ to $160 \mathrm{~ms}$, and the corrected QT interval was also prolonged from $370 \mathrm{~ms}$ to $400 \mathrm{~ms}$ (Fig. 3). VT of more than 3 beats was found three times and they lasted in total for 4 minutes and 10 seconds in the 15-minute period before amiodarone. Before discontinuation of amiodarone, VT was found to occur 5 times and lasted for 9 minutes and $40 \mathrm{sec}-$ onds in the 15-minute period.

We discontinued amiodarone, and administered $12 \mathrm{mg}$ of nifekalant, pure IKr blocker, over 5 minutes intravenously followed by a continuous dose of $9 \mathrm{mg}$ per hour. About 7 minutes after starting nifekalant, VT disappeared completely. QRS duration was not changed $(160 \mathrm{~ms})$, but the corrected
QT interval was markedly prolonged to $520 \mathrm{~ms}$.

Administration of sotalol ( $80 \mathrm{mg}$ per day) made it possible to withdraw nifekalant. Because her heart rate tended to be low, and an increase in $\beta$-blocker dosage was considered necessary, CRT-D implantation was conducted. After the increase of carvedilol to $10 \mathrm{mg}$ per day, she was discharged from our hospital.

\section{Discussion}

Several studies have demonstrated the usefulness of intravenous amiodarone and it is now recommended as a firstline drug for treatment of VT (5). While intravenous amiodarone is generally regarded as a safe treatment, there are several reports on proarrhythmia inducing TdP under certain conditions including electrolyte imbalance $(3,4,6,7)$. In the present case, incessant monomorphic VT, not TdP, was induced after injection of intravenous amiodarone. As far as we know, this is the first report on monomorphic VT induced after amiodarone infusion. Moreover, the amiodarone efficacy in the treatment of stable VT has not been fully elucidated (8-10).

The incessant VT in this patient, who had structural heart disease, maintained regular beats and was reproducibly induced by relatively short coupled PVC. VT always terminated and reappeared spontaneously and the VT cycle length was gradually prolonged (about 10\%) before spontaneous termination. These findings supported the reentrant mecha- 


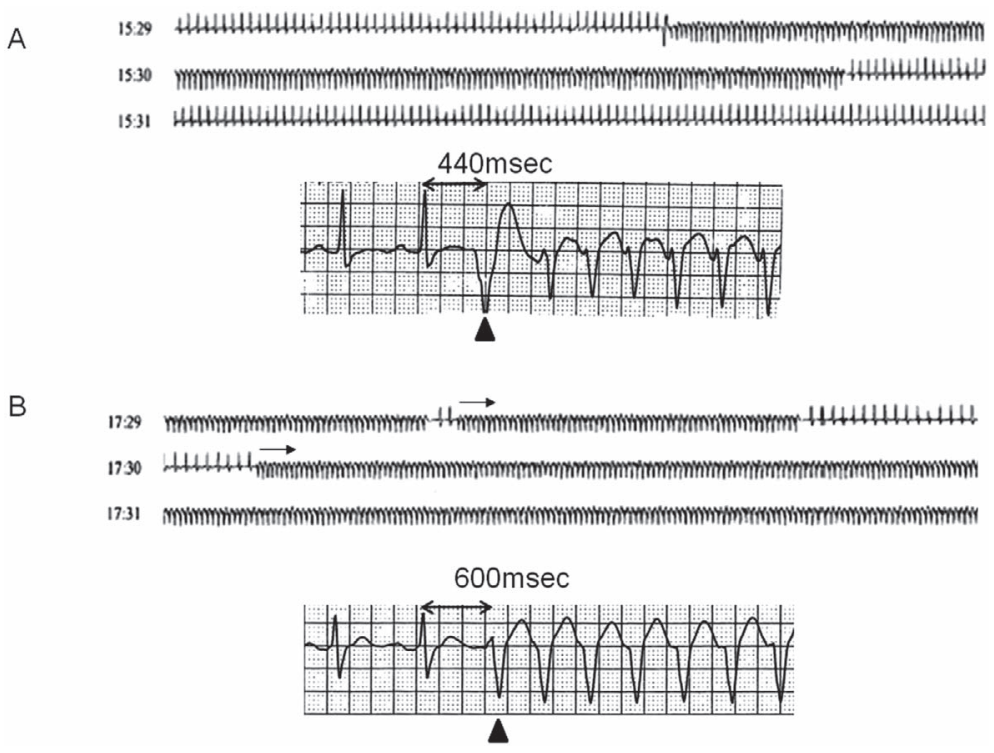

Figure 2. Recurrent ventricular tachycardia (VT) observed on the monitoring electrocardiogram (ECG) before and after amiodarone. Recurrent VT was suddenly observed on her monitoring ECG (A). After intravenous amiodarone, VT became more incessant and the duration of VT prolonged still more (B). Before amiodarone administration, relatively short coupled premature ventricular complex (PVC) (the coupling interval was $440 \mathrm{~ms}$ ), of which morphology was different from that of VT, induced recurrent VT (A). After amiodarone, VT was reproducibly triggered by PVC of which morphology was same as that of VT and the coupling interval of initiating PVC was relatively longer (600 ms) (B).

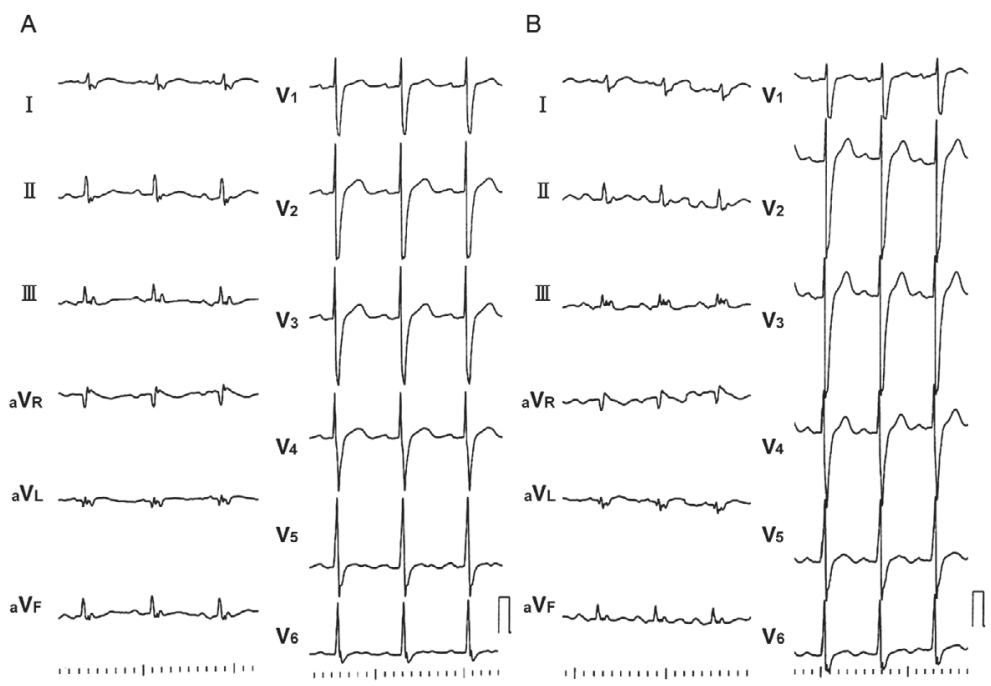

Figure 3. Twelve-lead electrocardiograms (ECGs) during sinus rhythm before and after amiodarone. Before amiodarone, QRS duration was $130 \mathrm{msec}$ and QTc interval was $370 \mathrm{msec}$ on the ECG (A). After amiodarone, QRS duration and QTc interval were prolonged to $160 \mathrm{msec}$ and $400 \mathrm{msec}$ (B).

\section{nism of this VT.}

Intravenous amiodarone inhibits sodium channels, inward L-type calcium channels, and has noncompetitive $\beta$ blockade effect, but the potassium channel blockade effect became more apparent after long-term therapy. It was possible that blockade of L-type calcium channels and $\beta$ blockade could suppress automaticity and triggered activity. However, in this case, the mechanism of VT was thought to be likely due to re-entry, these effects can just slow the VT rate. The slight prolongation of QT interval can be explained by potassium channel blockade effect of intravenous amiodarone. The prolongation of QRS duration, the decline of VT rate, and the change of QRS morphology of the initiating PVC after intravenous amiodarone suggested that the acute effect of amiodarone infusion mainly developed sodium channel blockade. The coupling interval between the 
A
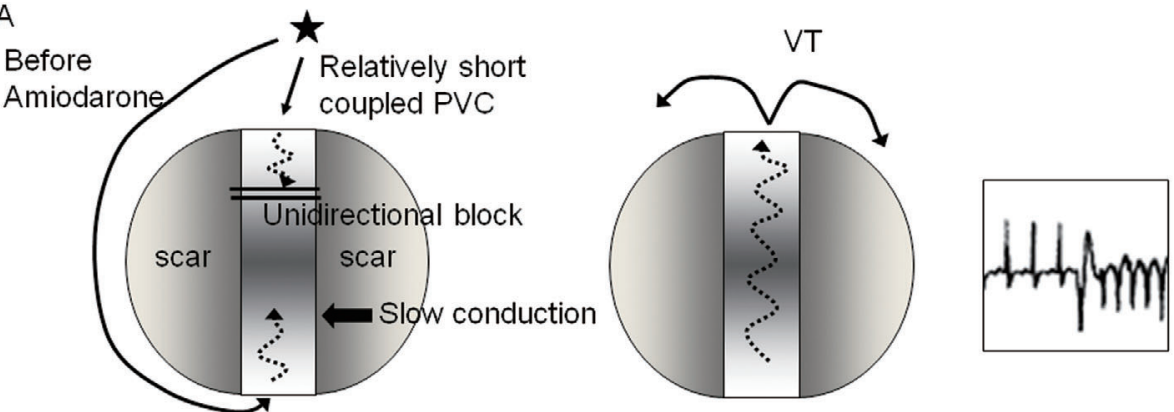

B Sinus rhythm
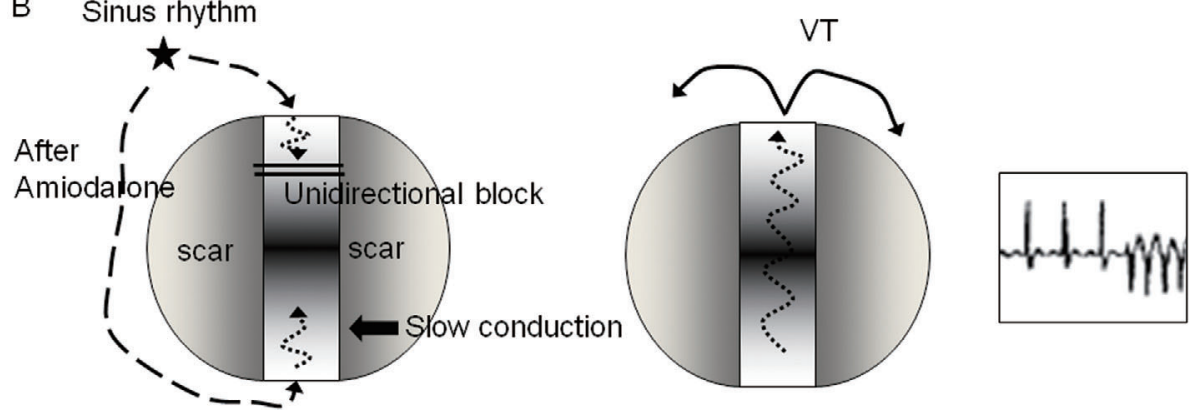

Figure 4. Illustration of postulated mechanism of ventricular tachycardia (VT) before and after amiodarone. Before amiodarone administration, relatively short coupled premature ventricular complex caused unidirectional block in critical slow pathway zone, and retrograde conduction formed the reentrant circuit resulting in recurrent VT (A). After amiodarone administration, its blocking effect of sodium channel could create the unidirectional block in the critical slow conduction zone even during sinus rhythm and induced the incessant monomorphic VT (B).

initiating PVC and the next beat of VT was nearly identical to VT cycle length.

We depicted the postulated mechanism of VT in this case as shown in Fig. 4. Sodium channel blockade of amiodarone was thought to create the unidirectional block in the critical slow conduction zone even during sinus rhythm and induced the incessant monomorphic VT as a proarrhythmia. At the time of admission, the morphology of occasional PVC was similar to that of documented incessant VT, and QRS duration was $150 \mathrm{~ms}$, this was relatively longer than the QRS duration before amiodarone. These findings suggested that a similar proarrhythmic situation had occurred in this patient at that time for some reasons but the difference between the coupling intervals of PVCs at the time of admission and after amiodarone determined whether the arrhythmias were sustained or not. Subsequent intravenous nifekalant, which promptly inhibits IKr channels and prolongs effective refractory period of ventricular myocardium in the critical slow pathway, could make VT disappeared completely. This successful suppression of VT could result from combination effects of amiodarone and nifekalant.

It was possible that amiodarone suppressed the original initiating PVC and accordingly the initiating PVC was changed. The effects of amiodarone other than sodium channel blockade could affect initiating PVC. But, nifekalant, $\mathrm{IKr}$ inhibitor, which has little effect on sodium and calcium channels, swept the initiating PVC away. This indicated that the initiating PVC was due to re-entrant mechanism rather than abnormal automaticity and triggered activity which de- pended on mainly intracellular sodium and calcium ion concentrations. In addition, (i) amiodarone usually suppressed the abnormal automaticity and triggered activity, (ii) a similar QRS prolongation was observed at the time of hospitalization and after amiodarone when similar PVC were found and VT worsened, (iii) the coupling interval between the initiating PVC and the preceding QRS was nearly constant, (iv) sustained VT was suppressed concomitant with the complete suppression of the PVCs after nifekalant administration. These also support our re-entrant hypothesis that sodium channel blockade affected on this patient. Furthermore, Duff et al demonstrated experimentally that sodium channel blocker precipitated monomorphic VT and its inducibility was suppressed by potassium channel blocker (11). Their experiment also supports our hypothesis. However, we could not completely exclude the possibility of initiating PVC from the exit-site of critical slow conduction after amiodarone. Initiating PVC from the exit-site could be analogous to the QRS morphology of VT and the coupling interval between the 1st and 2nd beat of VT could be similar to VT cycle length.

We encountered incessant monomorphic VT induced after injection of intravenous amiodarone. It is important to be aware of the proarrhythmic effect of amiodarone which may lead to an electrical storm of monomorphic VT.

The authors state that they have no Conflict of Interest (COI). 


\section{References}

1. Vassallo P, Trohman RG. Prescribing amiodarone. JAMA 298: 1312-1322, 2007.

2. Singh BN. Amiodarone: historical development and pharmacologic profile. Am Heart J 106: 788-797, 1983.

3. Hohnloser SH, Klingenheben T, Singh BN. Amiodaroneassociated proarrhythmic effects. A review with special reference to torsade de pointes tachycardia. Ann Intern Med 121: 529-535, 1994.

4. Brown MA, Smith WM, Lubbe WF, Norris RM. Amiodaroneinduced torsades de pointes. Eur Heart J 7: 234-239, 1986.

5. Goldschlager N, Epstein AE, Naccarelli GV, et al; Practice Guidelines Sub-committee, North American Society of Pacing and Electrophysiology (HRS). A practical guide for clinicians who treat patients with amiodarone: 2007. Heart Rhythm 4: 1250-1259, 2007.

6. Kusano KF, Hata Y, Yumoto A, Emori T, Sato T, Ohe T. Torsade de pointes with a normal QT interval associated with hypokalemia: a case report. Jpn Circ J 65: 757-760, 2001.
7. Geng DF, Jin DM, Wang JF, Luo YJ, Wu W. Clinical study of amiodarone-associated torsade de pointes in Chinese people. Pacing Clin Electrophysiol 29: 712-718, 2006.

8. Tomlinson DR, Cherian P, Betts TR, Bashir Y. Intravenous amiodarone for the pharmacological termination of haemodynamicallytolerated sustained ventricular tachycardia: is bolus dose amiodarone an appropriate first-line treatment? Emerg Med J 25: 15-18, 2008.

9. Marill KA, deSouza IS, Nishijima DK, Stair TO, Setnik GS, Ruskin JN. Amiodarone is poorly effective for the acute termination of ventricular tachycardia. Ann Emerg Med 47: 217-224, 2006.

10. Marill KA, deSouza IS, Nishijima DK, et al. Amiodarone or procainamide for the termination of sustained stable ventricular tachycardia: an historical multicenter comparison. Acad Emerg Med 17: 297-306, 2010.

11. Duff HJ, Stemler M, Thannhauser T, Laganiere S, Rude E, Lester W. Proarrhythmia of a class Ic drug: suppression by combination with a drug prolonging repolarization in the dog late after infarction. J Pharmacol Exp Ther 274: 508-515, 1995.

(C) 2011 The Japanese Society of Internal Medicine http://www.naika.or.jp/imindex.html 